\title{
A combinatorial derivation with Schröder paths of a determinant representation of Laurent biorthogonal polynomials
}

\author{
Shuhei Kamioka* \\ Department of Applied Mathematics and Physics, Graduate School of Informatics \\ Kyoto University, Kyoto 606-8501, Japan \\ kamioka@amp.i.kyoto-u.ac.jp
}

Submitted: Aug 28, 2007; Accepted: May 26, 2008; Published: May 31, 2008

Mathematics Subject Classifications: 05A15, 42C05, 05E35

\begin{abstract}
A combinatorial proof in terms of Schröder paths and other weighted plane paths is given for a determinant representation of Laurent biorthogonal polynomials (LBPs) and that of coefficients of their three-term recurrence equation. In this process, it is clarified that Toeplitz determinants of the moments of LBPs and their minors can be evaluated by enumerating certain kinds of configurations of Schröder paths in a plane.
\end{abstract}

\section{Introduction}

Laurent biorthogonal polynomials (LBPs) appeared in problems related to Thron type continued fractions (T-fractions), two-point Padé approximants and moment problems (see, e.g., [6]), and are studied by many authors (e.g. [6, 4, 5, 11, 10]). We recall fundamental properties of LBPs.

Notation remark. In this paper the symbols $i, j, k, K, m, n$ and $\ell$ are used for nonnegative integers and for integers, respectively. The symbol $\mathrm{X}_{a, b, \ldots, z}$ with multiple subscripts, if specifically undefined, denotes " $\mathrm{X}_{a}, \mathrm{X}_{b}, \ldots$ and $\mathrm{X}_{z}$."

Let $\mathbb{K}$ be a field. We call a sequence $\left(P_{n}(z)\right)_{n=0}^{\infty}$ a sequence of Laurent biorthogonal polynomials with respect to a linear functional $\mathcal{L}: \mathbb{K}\left[z^{-1}, z\right] \rightarrow \mathbb{K}$, if, for each $n \geq 0$, $P_{n}(z) \in \mathbb{K}[z]$ is a polynomial of degree $n$ which possesses the orthogonality property

$$
\mathcal{L}\left[z^{-\ell} P_{n}(z)\right] \begin{cases}=0, & 0 \leq \ell \leq n-1 \\ \neq 0, & \ell=n\end{cases}
$$

\footnotetext{
${ }^{*}$ JSPS Research Fellow.
} 
In this paper we normalize the 0 -th polynomial as $P_{0}(z)=1$ for simplicity. The LBPs $P_{n}(z)$ satisfy a three-term recurrence equation of the form

$$
P_{n+1}(z)=\left(\alpha_{n} z-\gamma_{n}\right) P_{n}(z)-\beta_{n} z P_{n-1}(z), \quad n \geq 1
$$

with $P_{0}(z)=1$ and $P_{1}(z)=\alpha_{0} z-\gamma_{0}$, where the coefficients $\alpha_{n}, \beta_{n}$ and $\gamma_{n}$ are some nonzero constants. The linear functional $\mathcal{L}$ is characterized by its moments

$$
\mu_{\ell}=\mathcal{L}\left[z^{\ell}\right], \quad \ell \in \mathbb{Z}
$$

Then we have the following theorem related to Toeplitz determinants of the moments.

Theorem 1. The sequence $\left(\mu_{\ell}\right)_{\ell=-\infty}^{\infty}$ of the moments of $\mathcal{L}$ is 1-regular, namely, it satisfies the condition that the Toeplitz determinants

$$
\Delta_{n}^{(0)}=\left|\begin{array}{cccc}
\mu_{0} & \mu_{1} & \cdots & \mu_{n-1} \\
\mu_{-1} & \mu_{0} & \cdots & \mu_{n-2} \\
\vdots & \vdots & & \vdots \\
\mu_{-n+1} & \mu_{-n+2} & \cdots & \mu_{0}
\end{array}\right|, \quad \Delta_{n}^{(1)}=\left|\begin{array}{cccc}
\mu_{1} & \mu_{2} & \cdots & \mu_{n} \\
\mu_{0} & \mu_{1} & \cdots & \mu_{n-1} \\
\vdots & \vdots & & \vdots \\
\mu_{-n+2} & \mu_{-n+3} & \cdots & \mu_{1}
\end{array}\right|
$$

are nonzero for every $n \geq 0$, where $\Delta_{0}^{(0)}=\Delta_{0}^{(1)}=1$. Moreover, the coefficients $\alpha_{n}, \beta_{n}$ and $\gamma_{n}$ of the recurrence equation (1) satisfy the equalities with the determinants

$$
\frac{\alpha_{n}}{\gamma_{n}}=\frac{\Delta_{n+1}^{(0)} \Delta_{n}^{(1)}}{\Delta_{n}^{(0)} \Delta_{n+1}^{(1)}}, \quad \frac{\beta_{n}}{\alpha_{n-1} \alpha_{n}}=-\frac{\Delta_{n-1}^{(0)} \Delta_{n+1}^{(1)}}{\Delta_{n}^{(0)} \Delta_{n}^{(1)}}, \quad \frac{\beta_{n}}{\gamma_{n-1} \gamma_{n}}=-\frac{\Delta_{n+1}^{(0)} \Delta_{n-1}^{(1)}}{\Delta_{n}^{(0)} \Delta_{n}^{(1)}},
$$

and the LBPs $P_{n}(z)$ have the determinant representation

$$
P_{n}(z)=\left(\prod_{k=0}^{n-1} \alpha_{k}\right)\left(\Delta_{n}^{(0)}\right)^{-1}\left|\begin{array}{cccc}
\mu_{0} & \mu_{1} & \cdots & \mu_{n} \\
\mu_{-1} & \mu_{0} & \cdots & \mu_{n-1} \\
\vdots & \vdots & & \vdots \\
\mu_{-n+1} & \mu_{-n+2} & \cdots & \mu_{1} \\
1 & z & \cdots & z^{n}
\end{array}\right| .
$$

Our aim in this paper is to present a combinatorial interpretation of LBPs and their properties. Especially we present to Theorem 1 a combinatorial proof in terms of Schröder paths and other weighted plane paths. This paper is organized as follows. In Section 2, we introduce and define several combinatorial concepts used throughout the paper: Schröder paths and Favard-LBP paths which are weighted. Particularly, following [7], we interpret the moments and the LBPs in terms of total weight of Schröder paths and that of FavardLBP paths, respectively. In Section 3, we evaluate the Toeplitz determinants and their minors by enumerating "non-intersecting" and "dense" configurations of Schröder paths (to be defined in there). In Section 4, we show a bijection between non-intersecting and dense configurations and Favard-LBP paths and clarify a correspondence between them. Finally, in Section 5, we give an immediate proof of Theorem 1. 
This combinatorial approach to orthogonal functions is due to Viennot [9]. He gave to general (ordinary) orthogonal polynomials, following Flajolet's interpretation [2] of Jacobi type continued fractions (J-fractions), a combinatorial interpretation in terms of Motzkin and Favard paths. Specifically he proved a claim for orthogonal polynomials similar to Theorem 1, for which he evaluated Hankel determinants of moments and their minors with non-intersecting configurations of Motzkin paths, and show a one-to-one correspondence, or a duality, between such a configuration and a Favard path.

\section{Combinatorial preliminaries}

In this paper we deal with paths on a simple directed graph, for which we use the following notation. The symbol $\left[v_{0}, \ldots, v_{\ell}\right], \ell \geq 0$, denotes the path going from $v_{0}$ to $v_{\ell}$ via $v_{1}, \ldots, v_{\ell-1}$, where $v_{i}$ are vertices. Particularly we call a path of the form $\left[v_{0}\right]$, consisting of one vertex and no edges, empty.

Weight of a finite graph is a fundamental concept in our combinatorial discussion. First we weight each of its vertices and edges by a map $w$ to $\mathbb{K}$. Then we do a finite graph $F$ by

$$
w(F)=\prod_{q \text { in } F} w(q)
$$

where the product is over all the vertices and edges in $F$. For example, a path weighs as

$$
w\left(\left[v_{0}, \ldots, v_{\ell}\right]\right)=\left(\prod_{i=0}^{\ell} w\left(v_{i}\right)\right)\left(\prod_{i=0}^{\ell-1} w\left(\left(v_{i}, v_{i+1}\right)\right)\right)
$$

where $\left(v_{i}, v_{i+1}\right)$ denotes the edge going from $v_{i}$ to $v_{i+1}$.

\subsection{Schröder paths and moments}

Commonly, as in [1], a Schröder path is defined as a lattice path from $(0,0)$ to $(n, n)$, $n \geq 0$, consisting of the three kinds of edges $(1,0),(0,1)$ and $(1,1)$ and not going above the line $\{x=y\}$. Such paths are counted by the large Schröder numbers (A006318 in [8]). In this paper, instead, we use the following definition for convenience.

Let $G=G^{-} \cup G^{+}$be the union of the two simple directed graphs $G^{-}=\left(V^{-}, E^{-}\right)$and $G^{+}=\left(V^{+}, E^{+}\right)$consisting of the vertices

$$
\begin{gathered}
V^{-}=\bigcup_{k=0}^{\infty} V_{k}^{-}, \quad V^{+}=\bigcup_{k=0}^{\infty} V_{k}^{+}, \\
V_{k}^{-}=\{(2 j+k, k) ; j \in \mathbb{Z}\}, \quad V_{k}^{+}=\{(2 j+k+1, k) ; j \in \mathbb{Z}\}
\end{gathered}
$$


and the edges

$$
\begin{aligned}
E^{-}=\bigcup_{k=0}^{\infty} U_{k}^{-} \cup \bigcup_{k=1}^{\infty} D_{k}^{-} \cup \bigcup_{k=0}^{\infty} H_{k}^{-}, \quad E^{+}=\bigcup_{k=0}^{\infty} U_{k}^{+} \cup \bigcup_{k=1}^{\infty} D_{k}^{+} \cup \bigcup_{k=0}^{\infty} H_{k}^{+}, \\
U_{k}^{-}=\left\{((j, k),(j-1, k+1)) \in V_{k}^{-} \times V_{k+1}^{-}\right\}, \\
D_{k}^{-}=\left\{((j, k),(j-1, k-1)) \in V_{k}^{-} \times V_{k-1}^{-}\right\} \\
H_{k}^{-}=\left\{((j, k),(j-2, k)) \in V_{k}^{-} \times V_{k}^{-}\right\} \\
U_{k}^{+}=\left\{((j, k),(j+1, k+1)) \in V_{k}^{+} \times V_{k+1}^{+}\right\} \\
D_{k}^{+}=\left\{((j, k),(j+1, k-1)) \in V_{k}^{+} \times V_{k-1}^{+}\right\}, \\
H_{k}^{+}=\left\{((j, k),(j+2, k)) \in V_{k}^{+} \times V_{k}^{+}\right\} .
\end{aligned}
$$

Then a Schröder path is such a path on the graph $G$ that both of its endpoints lie in $\{y=0\}$ (namely $\in V_{0}^{-} \cup V_{0}^{+}$). See Figure 1 for example. As in the figure, in this paper, we draw $G$ with thin lines, in which we do $G^{-}$and $G^{+}$with (black) solid lines and (red) dotted ones, respectively. Additionally we draw a vertex and an edge in a Schröder path with a small circle and a bold line segment, respectively, in which we draw those on $G^{-}$ with a (black) filled circle and a solid line segment while we do those on $G^{+}$with a (red) not filled one and a dotted one. Note that the graphs $G^{-}$and $G^{+}$are disjoint and that a path on $G^{-}$goes from right to left while that on $G^{+}$does from left to right.

We call edges in $U_{k}^{-} \cup U_{k}^{+}$, in $D_{k}^{-} \cup D_{k}^{+}$and in $H_{k}^{-} \cup H_{k}^{+}$up-diagonal, down-diagonal and horizontal, respectively. We assume that, when we focus the set $\{y=k\} \subset \mathbb{R}^{2}$, we may see the vertices in $V_{k}^{-} \cup V_{k}^{+}$, the horizontal edges in $H_{k}^{-} \cup H_{k}^{+}$and no more, while, when we focus $\{k<y<k+1\} \subset \mathbb{R}^{2}$, we may see the up-diagonal edges in $U_{k}^{-} \cup U_{k}^{+}$, the down-diagonal edges in $D_{k+1}^{-} \cup D_{k+1}^{+}$and no more. (Thus, for example, when we focus $\{k \leq y<k+1\} \subset$ $\mathbb{R}^{2}$, we may see the vertices in $V_{k}^{-} \cup V_{k}^{+}$, the edges in $\left(U_{k}^{-} \cup U_{k}^{+}\right) \cup\left(D_{k+1}^{-} \cup D_{k+1}^{+}\right) \cup\left(H_{k}^{-} \cup H_{k}^{+}\right)$ and no more.)

We order the vertices in $V_{0}^{-}$from left to right as

$$
(2 j, 0)<\left(2 j^{\prime}, 0\right) \text { on } V_{0}^{-} \Longleftrightarrow j<j^{\prime} \text { on } \mathbb{Z} \text {. }
$$

We call a Schröder path on $G^{-}$from $(2 j, 0)$ to $\left(2 j^{\prime}, 0\right)$ where $j \geq j^{\prime}$ or that on $G^{+}$from $(2 j+1,0)$ to $\left(2 j^{\prime}-1,0\right)$ where $j<j^{\prime}$ a path froward $(2 j, 0)$ toward $\left(2 j^{\prime}, 0\right)$. We use the symbol $\Omega_{-j+j^{\prime}}$ for the set of all the Schröder paths froward $(2 j, 0)$ toward $\left(2 j^{\prime}, 0\right)$ where

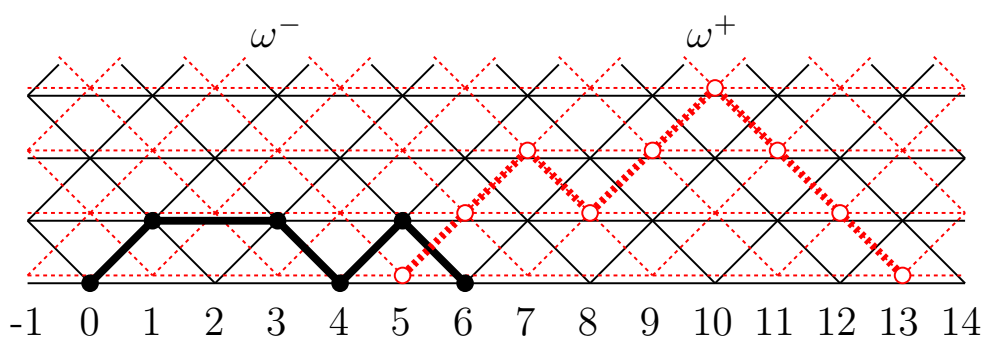

Figure 1: Schröder paths $\omega^{-}$(the left one on $G^{-}$) and $\omega^{+}$(the right one on $G^{+}$) on $G$. 
we identify two paths if they coincide by a translation in the horizontal ( $x$-axis) direction. For example, the paths in Figure 1 are classified as $\omega^{-} \in \Omega_{-3}$ and $\omega^{+} \in \Omega_{5}$, and the set $\Omega_{3}$ has the six paths in Figure 2 .

For a Schröder path $\omega$, by deleting its vertices and edges in $\{0 \leq y<1\}$ and then by translating the remaining by $(-1,-1)$, we obtain a set of Schröder paths, for which we use the symbol $r(\omega)$. For example, as in Figure 3, for the paths $\omega^{-}$and $\omega^{+}$in Figure $1, r\left(\omega^{-}\right)$and $r\left(\omega^{+}\right)$are sets of two and one paths, respectively. Moreover, for a set $\xi$ of Schröder paths, we set $r(\xi)=\cup_{\omega \in \xi} r(\omega)$. Then we clearly have the following.

Lemma 2. Let $\xi$ be a set of Schröder paths. Then the set $r(\xi)$ has a path froward $(2 j, 0)$ (resp. toward $(2 j, 0))$ if and only if $\xi$ has a path going through the square region $(2 j+1,2 j+2) \times(0,1)$ with an up-diagonal edge (resp. going through $(2 j, 2 j+1) \times(0,1)$ with a down-diagonal edge).

We weight a Schröder path by (3), where we do its vertices and edges, using the coefficients $\alpha_{n}, \beta_{n}$ and $\gamma_{n}$ of the recurrence equation (1), by

$$
w(q)=\left\{\begin{array} { l l } 
{ ( \gamma _ { k } ) ^ { - 1 } , } & { q \in V _ { k } ^ { - } , } \\
{ 1 , } & { q \in U _ { k } ^ { - } , } \\
{ \beta _ { k } , } & { q \in D _ { k } ^ { - } , } \\
{ \alpha _ { k } , } & { q \in H _ { k } ^ { - } , }
\end{array} \quad \left\{\begin{array}{ll}
\left(\alpha_{k}\right)^{-1}, & q \in V_{k}^{+}, \\
1, & q \in U_{k}^{+}, \\
\beta_{k}, & q \in D_{k}^{+}, \\
\gamma_{k}, & q \in H_{k}^{+} .
\end{array}\right.\right.
$$

For example, the paths in Figure 1 weigh as

$$
w\left(\omega^{-}\right)=\frac{\alpha_{1}\left(\beta_{1}\right)^{2}}{\left(\gamma_{0}\right)^{3}\left(\gamma_{1}\right)^{3}}, \quad w\left(\omega^{+}\right)=\frac{\beta_{1}\left(\beta_{2}\right)^{2} \beta_{3}}{\left(\alpha_{0}\right)^{2}\left(\alpha_{1}\right)^{3}\left(\alpha_{2}\right)^{3} \alpha_{3}} .
$$

We can equivalently rewrite the way (3) with (6) to weight a Schröder path into the edge-oriented way

$$
w(\omega)= \begin{cases}\left(\gamma_{0}\right)^{-1} \prod_{e \text { in } \omega} w(e) & \text { if } \omega \text { goes on } G^{-}, \\ \left(\alpha_{0}\right)^{-1} \prod_{e \text { in } \omega} w(e) & \text { if } \omega \text { goes on } G^{+}\end{cases}
$$

where the product is over all the edges in $\omega$, with

$$
w(e)=\left\{\begin{array} { l l } 
{ 1 , } & { e \in U _ { k } ^ { - } , } \\
{ \beta _ { k } ( \gamma _ { k - 1 } \gamma _ { k } ) ^ { - 1 } , } & { e \in D _ { k } ^ { - } , } \\
{ \alpha _ { k } ( \gamma _ { k } ) ^ { - 1 } , } & { e \in H _ { k } ^ { - } , }
\end{array} \quad \left\{\begin{array}{ll}
1, & e \in U_{k}^{+}, \\
\beta_{k}\left(\alpha_{k-1} \alpha_{k}\right)^{-1}, & e \in D_{k}^{+}, \\
\gamma_{k}\left(\alpha_{k}\right)^{-1}, & e \in H_{k}^{+} .
\end{array}\right.\right.
$$

Thus, since [7], we can interpret the moments $\mu_{\ell}$ in terms of Schröder paths.

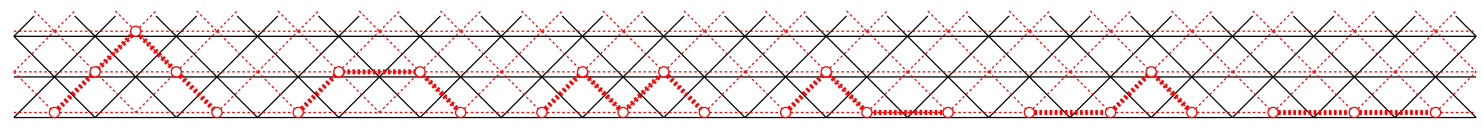

Figure 2: The Schröder paths in $\Omega_{3}$. 

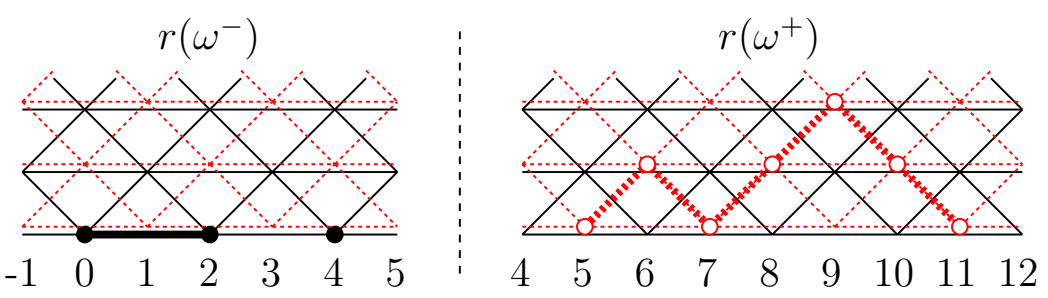

Figure 3: The sets $r\left(\omega^{-}\right)$and $r\left(\omega^{+}\right)$of Schröder paths obtained from the paths in Figure 1 .

Theorem 3. The moments $\mu_{\ell}$ of the functional $\mathcal{L}$ satisfy the equality with total weight of Schröder paths

$$
\frac{\mu_{\ell}}{\mu_{0}}=\gamma_{0} \sum_{\omega \in \Omega_{\ell}} w(\omega), \quad \ell \in \mathbb{Z}
$$

For example, a few of them are

$$
\begin{aligned}
\frac{\mu_{-2}}{\mu_{0}} & =\gamma_{0}\left[\frac{\beta_{1} \beta_{2}}{\left(\gamma_{0}\right)^{2}\left(\gamma_{1}\right)^{2} \gamma_{2}}+\frac{\alpha_{1} \beta_{1}}{\left(\gamma_{0}\right)^{2}\left(\gamma_{1}\right)^{2}}+\frac{\left(\beta_{1}\right)^{2}}{\left(\gamma_{0}\right)^{3}\left(\gamma_{1}\right)^{2}}+2 \frac{\alpha_{0} \beta_{1}}{\left(\gamma_{0}\right)^{3} \gamma_{1}}+\frac{\left(\alpha_{0}\right)^{2}}{\left(\gamma_{0}\right)^{3}}\right], \\
\frac{\mu_{-1}}{\mu_{0}} & =\gamma_{0}\left[\frac{\beta_{1}}{\left(\gamma_{0}\right)^{2} \gamma_{1}}+\frac{\alpha_{0}}{\left(\gamma_{0}\right)^{2}}\right] \\
\frac{\mu_{0}}{\mu_{0}} & =\gamma_{0} \cdot \frac{1}{\gamma_{0}} \\
\frac{\mu_{1}}{\mu_{0}} & =\gamma_{0} \cdot \frac{1}{\alpha_{0}} \\
\frac{\mu_{2}}{\mu_{0}} & =\gamma_{0}\left[\frac{\beta_{1}}{\left(\alpha_{0}\right)^{2} \alpha_{1}}+\frac{\gamma_{0}}{\left(\alpha_{0}\right)^{2}}\right], \\
\frac{\mu_{3}}{\mu_{0}} & =\gamma_{0}\left[\frac{\beta_{1} \beta_{2}}{\left(\alpha_{0}\right)^{2}\left(\alpha_{1}\right)^{2} \alpha_{2}}+\frac{\beta_{1} \gamma_{1}}{\left(\alpha_{0}\right)^{2}\left(\alpha_{1}\right)^{2}}+\frac{\left(\beta_{1}\right)^{2}}{\left(\alpha_{0}\right)^{3}\left(\alpha_{1}\right)^{2}}+2 \frac{\beta_{1} \gamma_{0}}{\left(\alpha_{0}\right)^{3} \alpha_{1}}+\frac{\left(\gamma_{0}\right)^{2}}{\left(\alpha_{0}\right)^{3}}\right] .
\end{aligned}
$$

Note that the total weight of Schröder paths in (7) is a generalization of the large Schröder number (A006318 in [8]), for it denotes the cardinality $\# \Omega_{\ell}$ when $\mathbb{K}=\mathbb{Q}$ and $\alpha_{n}=\beta_{n}=$ $\gamma_{n}=1$.

\subsection{Favard-LBP paths and LBPs}

Favard-LBP paths were introduced in [7], following Viennot's Favard paths for orthogonal polynomials [9], to combinatorially interpret LBPs, especially their recurrence equation, in which they are defined as paths from $\{y=0\}$ consisting of the three kinds of edges $(1,1)$, $(1,2)$ and $(0,1)$. In this paper, instead, we use the following definition for convenience. 
Let $G^{\mathrm{F}}=\left(V^{\mathrm{F}}, E^{\mathrm{F}}\right)$ be the simple directed graph consisting of the vertices

$$
V^{\mathrm{F}}=\bigcup_{k=0}^{\infty} V_{k}^{\mathrm{F}}, \quad V_{k}^{\mathrm{F}}=\{(2 j+k-1 / 2, k-1 / 2) ; j \in \mathbb{Z}\}
$$

and the edges

$$
\begin{gathered}
E^{\mathrm{F}}=\bigcup_{k=0}^{\infty} L_{k}^{\mathrm{F}} \cup \bigcup_{k=0}^{\infty} R_{k}^{\mathrm{F}} \cup \bigcup_{k=1}^{\infty} U_{k}^{\mathrm{F}}, \\
L_{k}^{\mathrm{F}}=\left\{((j, k),(j-1, k+1)) \in V_{k}^{\mathrm{F}} \times V_{k+1}^{\mathrm{F}}\right\}, \\
R_{k}^{\mathrm{F}}=\left\{((j, k),(j+1, k+1)) \in V_{k}^{\mathrm{F}} \times V_{k+1}^{\mathrm{F}}\right\}, \\
U_{k}^{\mathrm{F}}=\left\{((j, k-1),(j, k+1)) \in V_{k-1}^{\mathrm{F}} \times V_{k+1}^{\mathrm{F}}\right\} .
\end{gathered}
$$

Then a Favard- $L B P$ path is a path on the graph $G^{\mathrm{F}}$ which starts in $\{y=-1 / 2\}$ (namely, whose first vertex belongs to $V_{0}^{\mathrm{F}}$ ). See Figure 4 for example. As in the figure, in this paper we draw a vertex and an edge in a Favard-LBP path with a (blue) small triangle and a dashed line segment, respectively.

We use the symbol $\Omega_{n, i}^{\mathrm{F}}$, where $n \geq 0$ and $0 \leq i \leq n$, for the set of all the Favard-LBP paths going from $(2 i-1 / 2,-1 / 2)$ to $(n-1 / 2, n-1 / 2)$.

We weight a Favard-LBP path by (3), where we do its vertices and edges, using the coefficients $\alpha_{n}, \beta_{n}$ and $\gamma_{n}$ of the recurrence equation (1), by

$$
w(q)= \begin{cases}1, & q \in V^{\mathrm{F}} \\ \alpha_{k}, & q \in L_{k}^{\mathrm{F}}, \\ \gamma_{k}, & q \in R_{k}^{\mathrm{F}}, \\ \beta_{k}, & q \in U_{k}^{\mathrm{F}}\end{cases}
$$

For example, the paths in Figure 4 weigh as

$$
w\left(\omega_{1}^{\mathrm{F}}\right)=\gamma_{0} \gamma_{1} \alpha_{2} \gamma_{3}, \quad w\left(\omega_{2}^{\mathrm{F}}\right)=\gamma_{0} \beta_{2} \gamma_{3} \alpha_{4}, \quad w\left(\omega_{3}^{\mathrm{F}}\right)=\alpha_{0} \alpha_{1} \beta_{3} \gamma_{4} .
$$

As in [7] we can interpret the LBPs $P_{n}(z)$ in terms of Favard-LBP paths.

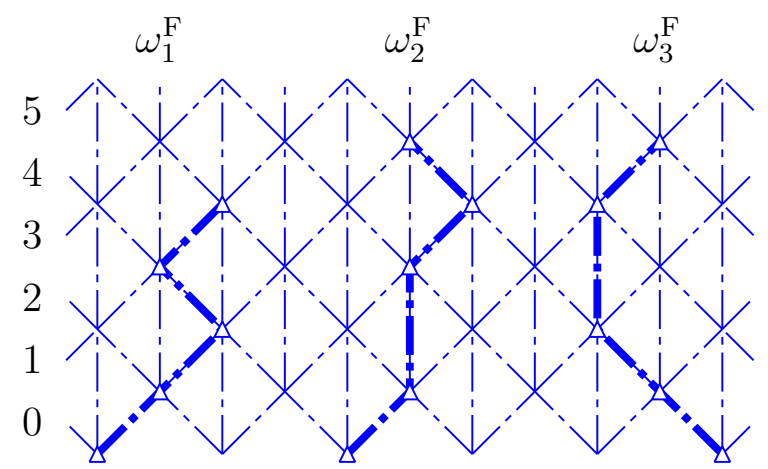

Figure 4: Favard-LBP paths $\omega_{1}^{\mathrm{F}}$ (left), $\omega_{2}^{\mathrm{F}}$ (middle) and $\omega_{3}^{\mathrm{F}}$ (right). 
Theorem 4. The LBPs $P_{n}(z)$ which satisfy the recurrence equation (1) are represented in terms of Favard-LBP paths as

$$
P_{n}(z)=\sum_{i=0}^{n}(-1)^{n-i} z^{i}\left(\sum_{\omega^{\mathrm{F}} \in \Omega_{n, i}^{\mathrm{F}}} w\left(\omega^{\mathrm{F}}\right)\right), \quad n \geq 0 .
$$

\section{Configurations of Schröder paths and Toeplitz de- terminants of moments}

Let us consider the determinant $\Delta_{n, i}$, where $n \geq 0$ and $0 \leq i \leq n$, of the moments $\mu_{\ell}$

$$
\Delta_{n, i}=\left|\begin{array}{cccccc}
\mu_{0} & \cdots & \mu_{i-1} & \mu_{i+1} & \cdots & \mu_{n} \\
\mu_{-1} & \cdots & \mu_{i-2} & \mu_{i} & \cdots & \mu_{n-1} \\
\vdots & & \vdots & \vdots & & \vdots \\
\mu_{-n+1} & \cdots & \mu_{-n+i} & \mu_{-n+i+2} & \cdots & \mu_{1}
\end{array}\right|
$$

obtained from the Toeplitz determinant $\Delta_{n+1}^{(0)}$ by deleting the last row and the column whose first element is $\mu_{i}$, where $\Delta_{0,0}=1$. We have through the permutation expansion with Theorem 3

$$
\Delta_{n, i}=\left(\mu_{0} \gamma_{0}\right)^{n} \sum_{\sigma} \sum_{\left(\omega_{j}\right)_{j=0}^{n-1}} \operatorname{sgn}(\sigma) \prod_{j=0}^{n-1} w\left(\omega_{j}\right)
$$

where the first sum is over all the bijections $\sigma:\{0, \ldots, n-1\} \rightarrow\{0, \ldots, n\} \backslash\{i\}$, the second sum is over all of such $n$-tuples $\left(\omega_{j}\right)_{j=0}^{n-1}$ of Schröder paths that $\omega_{j} \in \Omega_{-j+\sigma(j)}$,

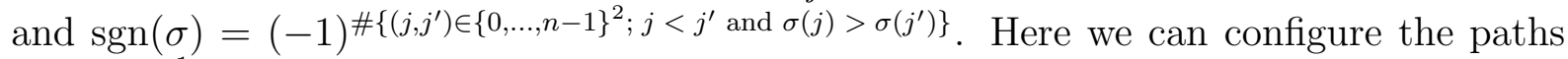
in $\left(\omega_{j}\right)_{j=0}^{n-1}$ on the graph $G$ so that $\omega_{j}$ goes froward $(2 j, 0)$ toward $(2 \sigma(j), 0)$ for each $0 \leq j \leq n$. Thus, in this section, we try to evaluate the determinant $\Delta_{n, i}$ by enumerating such configurations of Schröder paths.

\subsection{Configurations of Schröder paths}

First we give a formal definition of a configuration of Schröder paths. Let $S$ and $T$ be such two finite subsets of $V_{0}^{-}$that $\# S=\# T=n \geq 0$ and $\min S=(0,0)$ if $n \geq 1$. (The order on $V_{0}^{-}$is defined in (5).) Then a configuration of Schröder paths with sources $S$ and sinks $T$ is such a set of $n$ Schröder paths that exactly one path starts froward $s$ for each $s \in S$ and exactly one path ends toward $t$ for each $t \in T$. See Figure 5 for example. We use the symbol $\Xi(S, T)$ for the set of all such configurations.

A configuration $\xi \in \Xi(S, T)$ of Schröder paths induces such a bijection $\sigma_{\xi}: S \rightarrow T$ that $\xi$ has a Schröder path froward $s$ toward $\sigma_{\xi}(s)$ for each $s \in S$. We define a signature of the bijection in terms of its inversions by

$$
\operatorname{sgn}\left(\sigma_{\xi}\right)=(-1)^{\#\left\{\left(v, v^{\prime}\right) \in S^{2} ; v<v^{\prime} \text { and } \sigma_{\xi}(v)>\sigma_{\xi}\left(v^{\prime}\right)\right\}} .
$$




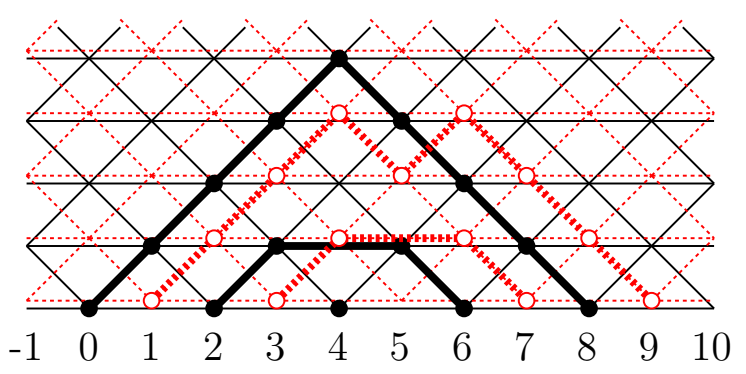

Figure 5: A configuration of Schröder paths with sources $\{(2 j, 0) ; j=0,1,2,3,4\}$ and sinks $\{(2 j, 0) ; j=0,1,2,4,5\}$.

For example, the configuration in Figure 5, letting it be $\xi$, induces the monotone decreasing bijection $\sigma_{\xi}((0,0))=(10,0), \sigma_{\xi}((2,0))=(8,0), \sigma_{\xi}((4,0))=(4,0), \sigma_{\xi}((6,0))=(2,0)$ and $\sigma_{\xi}((8,0))=(0,0)$.

\subsection{A combinatorial representation of determinants of moments in terms of configurations of Schröder paths}

We may evaluate the right hand side of (10) by enumerating all the configurations of Schröder paths in $\Xi\left(S_{n}, T_{n, i}\right)$, where $n \geq 0$ and $0 \leq i \leq n$, with the sources and sinks

$$
S_{n}=\{(2 j, 0) ; 0 \leq j \leq n-1\}, \quad T_{n, i}=\{(2 j, 0) ; 0 \leq j \leq n\} \backslash\{(2 i, 0)\},
$$

where $S_{0}$ and $T_{0,0}$ are the empty sets.

Let $\xi \in \Xi\left(S_{n}, T_{n, i}\right)$. It is contained in the region

$$
H_{n}=\{x-y>-1\} \cap\{x+y<2 n\},
$$

and we draw its border with (green) dashed lines for simplicity. On $\xi$, we call a square region $(2 i-1,2 i) \times(-1,0)$ or that $(j, j+1) \times(k, k+1) \subset\{0 \leq y<n\} \cap H_{n}$ through which no paths in $\xi$ go a sparse square, and draw its border with (blue) solid line segments. See Figure 6 for example. We assume that, when we focus $\{y=k\} \cap H_{n}$, we may see the top sides of sparse squares in $\{k-1<y<k\} \cap H_{n}$, the bottom ones of those in $\{k<y<k+1\} \cap H_{n}$ and no more, while, when we focus that in $\{k<y<k+1\} \cap H_{n}$, we may see the left and right sides of those in $\{k<y<k+1\} \cap H_{n}$ and no more.

Thus we can rewrite the equality (10) into

$$
\Delta_{n, i}=\left(\mu_{0} \gamma_{0}\right)^{n} \sum_{\xi \in \Xi\left(S_{n}, T_{n, i}\right)} \operatorname{sgn}\left(\sigma_{\xi}\right) w(\xi) .
$$

We may evaluate $\Delta_{n, i}$ more strictly.

Theorem 5. The determinant $\Delta_{n, i}$, where $n \geq 0$ and $0 \leq i \leq n$, of the moments $\mu_{\ell}$ is expanded with configurations of Schröder paths as

$$
\Delta_{n, i}=(-1)^{\frac{n(n-1)}{2}}\left(\mu_{0} \gamma_{0}\right)^{n} \sum_{\xi \in \tilde{\Xi}\left(S_{n}, T_{n, i}\right)} w(\xi),
$$




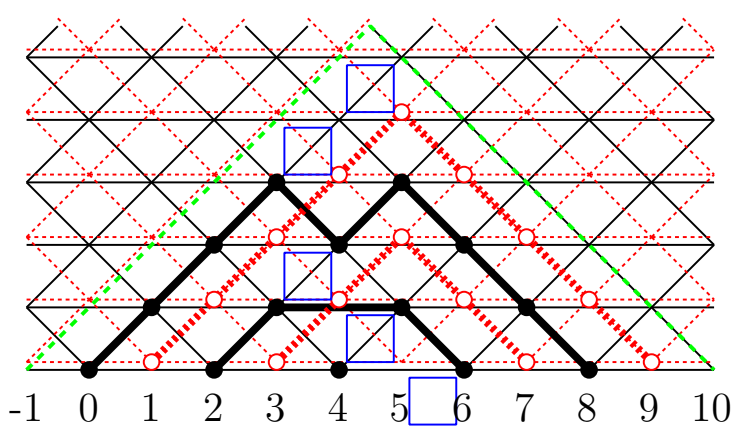

Figure 6: A configuration of Schröder paths in $\Xi\left(S_{5}, T_{5,3}\right)$ contained in $H_{5}$, and the sparse squares on it.

where $\widetilde{\Xi}\left(S_{n}, T_{n, i}\right)$ is the set of all the non-intersecting and dense configurations in $\xi \in$ $\Xi\left(S_{n}, T_{n, i}\right)$.

Here the terms "non-intersecting" and "dense" are defined as follows. We call a configuration of Schröder paths non-intersecting if it has no vertices shared by its two or more paths, and do intersecting if it is not non-intersecting. We call a configuration of Schröder paths in $\Xi\left(S_{n}, T_{n, i}\right)$ dense in $\{0 \leq y<K\} \cap H_{n}$ if we have at most one sparse square in $\{k \leq y<k+1\} \cap H_{n}$ for each $0 \leq k \leq K-1$, and do sparse in $\{K \leq y<K+1\} \cap H_{n}$ if we have two or more sparse squares in there. Then we call a configuration in $\Xi\left(S_{n}, T_{n, i}\right)$ dense if it is dense in $\{0 \leq y<n-1\} \cap H_{n}$, and do sparse if it is not dense. For example, in Figure 7, the left configuration is intersecting and dense while the right one is nonintersecting and sparse, for the left has a vertex at $(4,2)$ shared by its two paths and the right has three sparse squares in $\{3 \leq y<4\} \cap H_{5}$. On the other hand, the configuration in Figure 6 is non-intersecting and dense. We use the symbol $\Xi^{\prime}(S, T)$ for the set of all the non-intersecting configurations in $\Xi(S, T)$.

In the case $n=0$, the theorem clearly holds since the set $\widetilde{\Xi}\left(S_{0}, T_{0,0}\right)$ has the unique configuration of no paths which weighs 1 . Thus we assume $n \geq 1$ in the rest of this section.
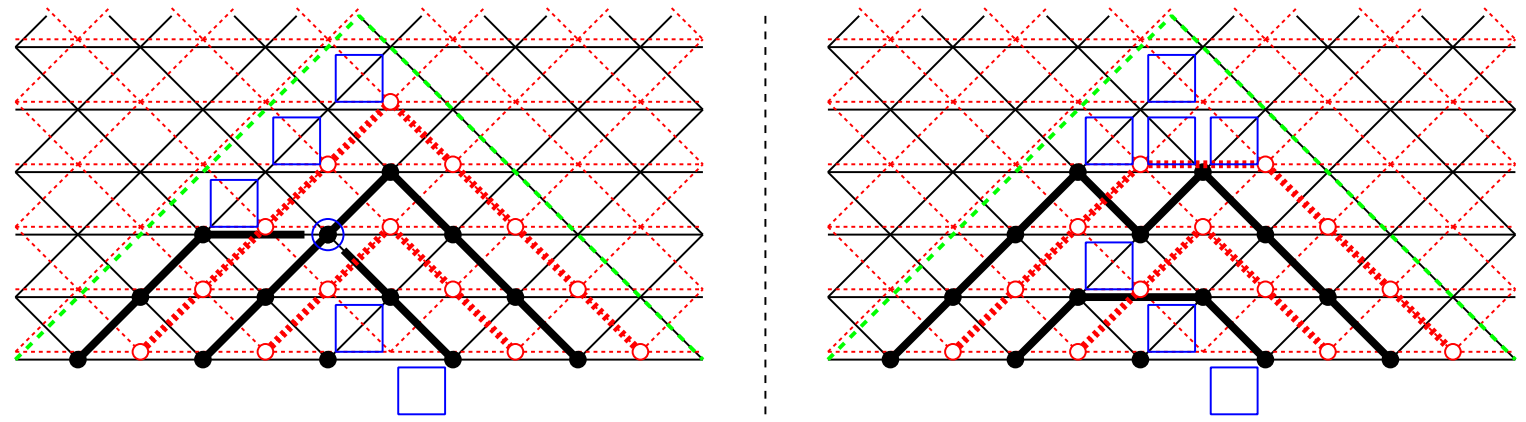

Figure 7: An intersecting configuration (left) and a sparse one (right) in $\Xi\left(S_{5}, T_{5,3}\right)$. 
Using the Gessel-Viennot methodology [3] we have

$$
\sum_{\xi \in \Xi\left(S_{n}, T_{n, i}\right) \backslash \Xi^{\prime}\left(S_{n}, T_{n, i}\right)} \operatorname{sgn}\left(\sigma_{\xi}\right) w(\xi)=0,
$$

since there exists an involution $\varphi$ on $\Xi\left(S_{n}, T_{n, i}\right) \backslash \Xi^{\prime}\left(S_{n}, T_{n, i}\right)$ of intersecting configurations satisfying for each $\xi \in \Xi\left(S_{n}, T_{n, i}\right) \backslash \Xi^{\prime}\left(S_{n}, T_{n, i}\right)$

$$
\operatorname{sgn}\left(\sigma_{\xi}\right)=-\operatorname{sgn}\left(\sigma_{\varphi(\xi)}\right), \quad w(\xi)=w(\varphi(\xi))
$$

Hence the following two lemmas shall validate the theorem. First we will extend the involution $\varphi$ into $\Xi^{\prime}\left(S_{n}, T_{n, i}\right) \backslash \widetilde{\Xi}\left(S_{n}, T_{n, i}\right)$.

Lemma 6. There exists an involution $\varphi$ on $\Xi^{\prime}\left(S_{n}, T_{n, i}\right) \backslash \widetilde{\Xi}\left(S_{n}, T_{n, i}\right)$ of non-intersecting but sparse configurations which satisfies the equalities (12) for each $\xi \in \Xi^{\prime}\left(S_{n}, T_{n, i}\right) \backslash$ $\widetilde{\Xi}\left(S_{n}, T_{n, i}\right)$.

Second we will explicitly determine the bijection $\sigma_{\xi}$ induced from a configuration $\xi$.

Lemma 7. For every non-intersecting and dense configuration $\xi \in \widetilde{\Xi}\left(S_{n}, T_{n, i}\right)$ the bijection $\sigma_{\xi}: S_{n} \rightarrow T_{n, i}$ is monotone decreasing.

The rest of this section is devoted to prove these two lemmas.

\subsection{Pieces of a non-intersecting configuration}

Let $\xi \in \Xi^{\prime}\left(S_{n}, T_{n, i}\right)$ be a non-intersecting configuration of Schröder paths, where $n \geq 1$ and $0 \leq i \leq n$. We call what we see when we look at $\xi$ through a window of the form $\left(\left[j, j^{\prime}\right] \times[k, k+1)\right) \cap H_{n}, j<j^{\prime}$, a piece of a configuration. We may construct a complete configuration by putting pieces as they fit together. Our first step to prove Lemmas 6 and 7 is to clarify what pieces we can have. For two pieces $p$ and $q$, if they fit together, we represent as $p q$ the piece obtained by gluing the right side of $p$ and the left side of $q$.

Proposition 8. Let $\xi \in \Xi^{\prime}\left(S_{n}, T_{n, i}\right)$ be a non-intersecting configuration of Schröder paths which is dense in $\{0 \leq y<K\} \cap H_{n}$, where $n \geq 1,0 \leq i \leq n$ and $0 \leq K \leq n-1$. Then its portion in $\{0 \leq y<K+1\} \cap H_{n}$ consists of the pieces $p_{1,2,2^{\prime}, 3,4,4^{\prime}, \mathrm{U}, \mathrm{U}^{\prime}, \mathrm{L} 1, \mathrm{~L} 2, \mathrm{~L} 2^{\prime}, \mathrm{R} 1, \mathrm{R} 1^{\prime}, \mathrm{R} 2}$ and $p_{\mathrm{S} 1, \ldots, \mathrm{S} 6}$ in Figure 8. More precisely

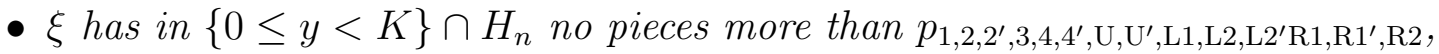

- $\xi$ is sparse in $\{K \leq y<K+1\} \cap H_{n}$ if and only if it has in there at least one piece of $p_{\mathrm{S} 1, \ldots, \mathrm{S} 6}$, and

- $\xi$ has $p_{\mathrm{U}^{\prime}}$ in $[2 j+k, 2 j+k+1] \times[k, k+1)$ if and only if it has $p_{\mathrm{U}}$ in $[2 j+k, 2 j+$ $k+1] \times[k-1, k)$.

This proposition follows the next three claims. 


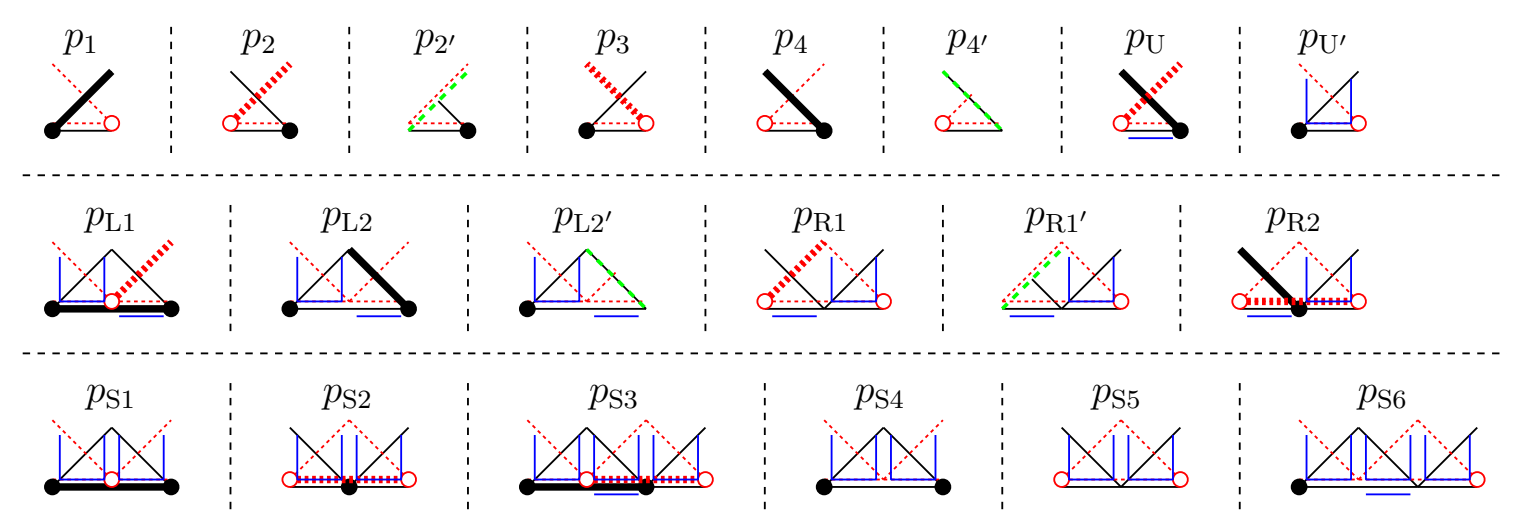

Figure 8: Pieces of a non-intersecting configuration of Schröder paths.

Claim 9. The portion of $\xi$ in $\{0 \leq y<1\} \cap H_{n}$ consists of $p_{1,2,2^{\prime}, 3,4,4^{\prime}, \mathrm{U}, \mathrm{L} 1, \mathrm{~L} 2, \mathrm{~L} 2^{\prime}, \mathrm{R} 1, \mathrm{R} 1^{\prime}, \mathrm{R} 2}$ and $p_{\mathrm{S} 1, \ldots, \mathrm{S} 6}$, where $\xi$ is sparse in there if and only if it has at least one of $p_{\mathrm{S} 1, \ldots, \mathrm{S} 6}$.

Proof. When we look at $\xi$ through $[2 j, 2 j+1] \times[0,1), 0 \leq j \leq n-1$, we see either $(2 j, 0) \in V_{0}^{-}$or $(2 j+1,0) \in V_{0}^{+}$with no edge connecting it, since $\xi$ has exactly one path froward $(2 j, 0)$. Thus we see any of the top six in Figure 9. When we look at $\xi$ through $([2 j-1,2 j] \times[0,1)) \cap H_{n}, 0 \leq j \leq n$, we should have a piece which fits that in $[2 j-2,2 j-1] \times[0,1)$ if $j \geq 1$ and that in $[2 j, 2 j+2] \times[0,1)$ if $j \leq n-1$, where we cannot have the one only of the vertex $(2 j-1,0) \in V_{0}^{+}$and $(2 j, 0) \in V_{0}^{-}$since $\xi$ has at most one path toward $(2 j, 0)$. Thus we see any of the lower seventeen in Figure 9. Then, after gluing two pieces as they share a horizontal edge or a bottom corner without a vertex, we obtain the pieces in Figure 8, except $p_{\mathrm{U}^{\prime}}$, and no more. The latter of the claim comes from the fact that every two of $p_{\mathrm{L} 1, \mathrm{~L} 2, \mathrm{~L} 2^{\prime}, \mathrm{R} 1, \mathrm{R} 1^{\prime}, \mathrm{R} 2}$ do not go together, for we have only one sparse square $(2 i-1,2 i) \times(-1,0)$ in $\{-1 \leq y<0\} \cap H_{n}$.

Claim 10. Suppose that $\xi$ is dense in $\{0 \leq y<1\} \cap H_{n}$ and has $p_{\mathrm{U}}$ in $[2 i-1,2 i] \times[0,1)$. Then the portion of $\xi$ in $\{1 \leq y<2\} \cap H_{n}$ consists of $p_{\mathrm{U}^{\prime}}$ in $[2 i-1,2 i] \times[1,2), p_{1,2,2^{\prime}, 3,4,4^{\prime}}$ and $p_{\mathrm{S} 1, \mathrm{~S} 2, \mathrm{~S} 4, \mathrm{~S} 5}$, where $\xi$ is sparse in there if and only if it has at least one of $p_{\mathrm{S} 1, \mathrm{~S} 2, \mathrm{~S} 4, \mathrm{~S} 5}$.

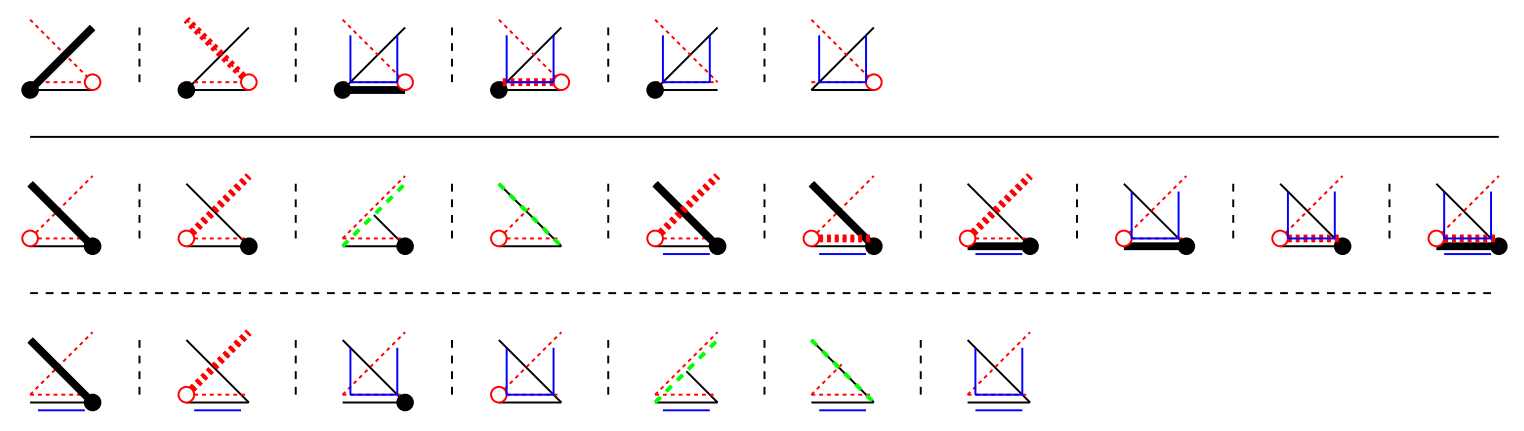

Figure 9: Possible pieces (before gluing). 
Proof. Since Claim 9, $\xi$ has in $\{0 \leq y<1\} \cap H_{n}$ no more than $p_{1,2,2^{\prime}, 3,4,4^{\prime}, \mathrm{U}}$. Thus, for every square region $(j, j+1) \times(0,1), 0 \leq j \leq 2 n-2$, $\xi$ has exactly one path which diagonally goes through there, except $[2 i-1,2 i] \times[0,1)$ for which it has exactly two such paths. Hence, since Lemma $2, r(\xi)$ is such a set of paths that exactly one path starts froward each $s \in\{(2 j, 0) ; 0 \leq j \leq n-2\} \backslash(2 i-2,0)$, exactly two start froward $(2 i-2,0)$ and exactly one ends toward each $t \in\{(2 j, 0) ; 0 \leq j \leq n-1\}$. Then, with this fact, we may prove the claim as we did Claim 9.

In a similar way, since Claims 9 and 10 with Lemma 2, we have the following.

Claim 11. Suppose that $\xi$ is dense in $\{0 \leq y<1\} \cap H_{n}$. Then $\xi$ has in there any of $p_{\mathrm{L} 1, \mathrm{~L} 2, \mathrm{~L} 2^{\prime}, \mathrm{R} 1, \mathrm{R} 1^{\prime}, \mathrm{R} 2, \mathrm{U}}$ as includes the top side of the border of the sparse square $(2 i-1,2 i) \times$ $(-1,0)$, and

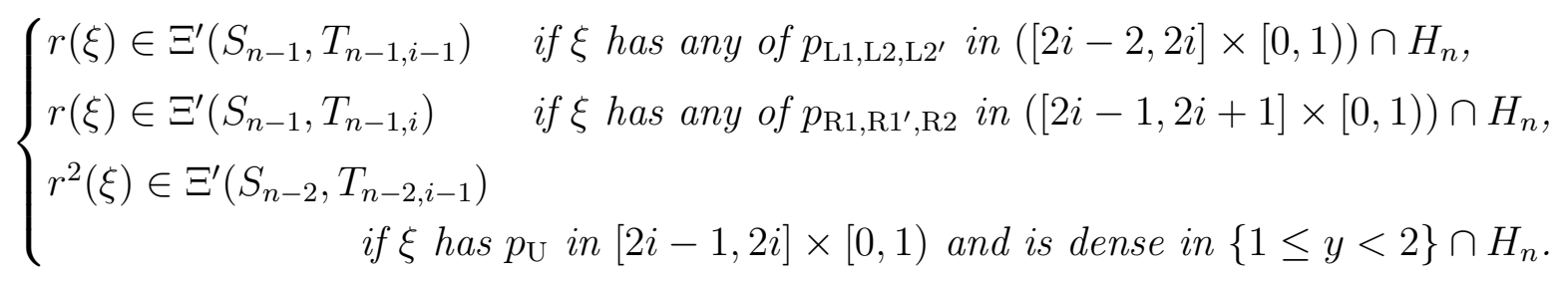

We obtain Proposition 8 by using these claims recursively.

\subsection{An involution for non-intersecting but sparse configurations}

Using Proposition 8, we may construct an involution $\varphi$ for non-intersecting but sparse configurations of Schröder paths which satisfies the equalities (12), and prove Lemma 6 . In the case $n=1$, a non-intersecting configuration in $\Xi^{\prime}\left(S_{1}, T_{1, i}\right), 0 \leq i \leq 1$, cannot have any piece of $p_{\mathrm{S} 1, \ldots, \mathrm{S} 6}$ in Figure 8 since they need at least two paths. Thus, since Proposition 8 , it is dense, that makes the lemma trivial. Hence we assume $n \geq 2$ in this subsection.

Let $\xi \in \Xi^{\prime}\left(S_{n}, T_{n, i}\right) \backslash \widetilde{\Xi}\left(S_{n}, T_{n, i}\right)$ be a non-intersecting configuration which is dense in $\{0 \leq y<K\} \cap H_{n}$ but is sparse in $\{K \leq y<K+1\} \cap H_{n}$, where $n \geq 2,0 \leq i \leq n$ and $0 \leq K \leq n-2$. Since Proposition 8 , $\xi$ has in $\{K \leq y<K+1\} \cap H_{n}$ at least one piece of $p_{\mathrm{S} 1, \ldots, \mathrm{S} 6}$ in Figure 8. Moreover the proposition tells us the following.

Claim 12. If $K \geq 1$, for each piece $p_{\mathrm{S} \nu}$ in $\{K \leq y<K+1\} \cap H_{n}$, $\xi$ has a portion whose form is any of $p p_{1, \ldots, 14}^{\mathrm{S}}$ in Figure 10 as contains the piece.

Here everything is ready to construct an involution.

Definition 1 (Involution $\varphi$ on $\Xi^{\prime}\left(S_{n}, T_{n, i}\right) \backslash \widetilde{\Xi}\left(S_{n}, T_{n, i}\right)$ ). Let $n \geq 2$ and $0 \leq i \leq n$. For a given configuration $\xi \in \Xi^{\prime}\left(S_{n}, T_{n, i}\right) \backslash \widetilde{\Xi}\left(S_{n}, T_{n, i}\right)$ of Schröder paths, which is dense in $\{0 \leq y<K\} \cap H_{n}$ but is sparse in $\{K \leq y<K+1\} \cap H_{n}, 0 \leq K \leq n-2$, we make a configuration $\varphi(\xi) \in \Xi^{\prime}\left(S_{n}, T_{n, i}\right) \backslash \widetilde{\Xi}\left(S_{n}, T_{n, i}\right)$, referring Figures 8, 10 and 11 , as follows. Let $\square \subset \mathbb{R}^{2}$ be the leftmost set which minimally contains a piece of $\xi$ in $\{K \leq y<K+1\} \cap H_{n}$ whose form is any of $p_{\mathrm{S} 1, \ldots, \mathrm{S} 6}$. 


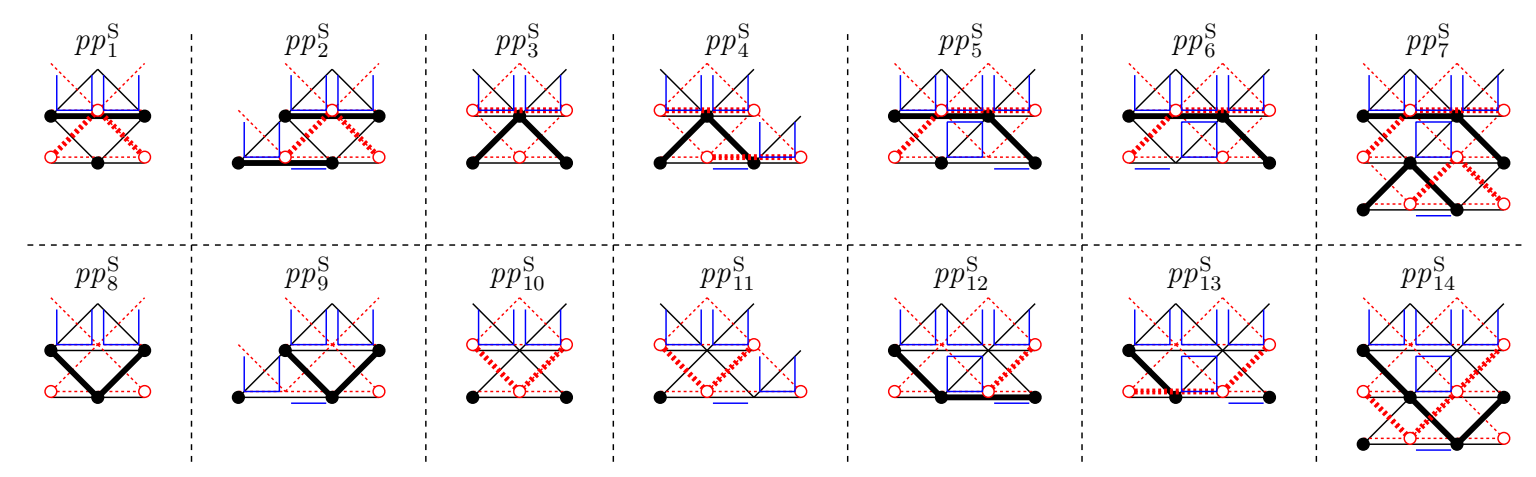

Figure 10: Portions containing any of the pieces $p_{\mathrm{S} 1, \ldots, \mathrm{S} 6}$ in Figure 8.

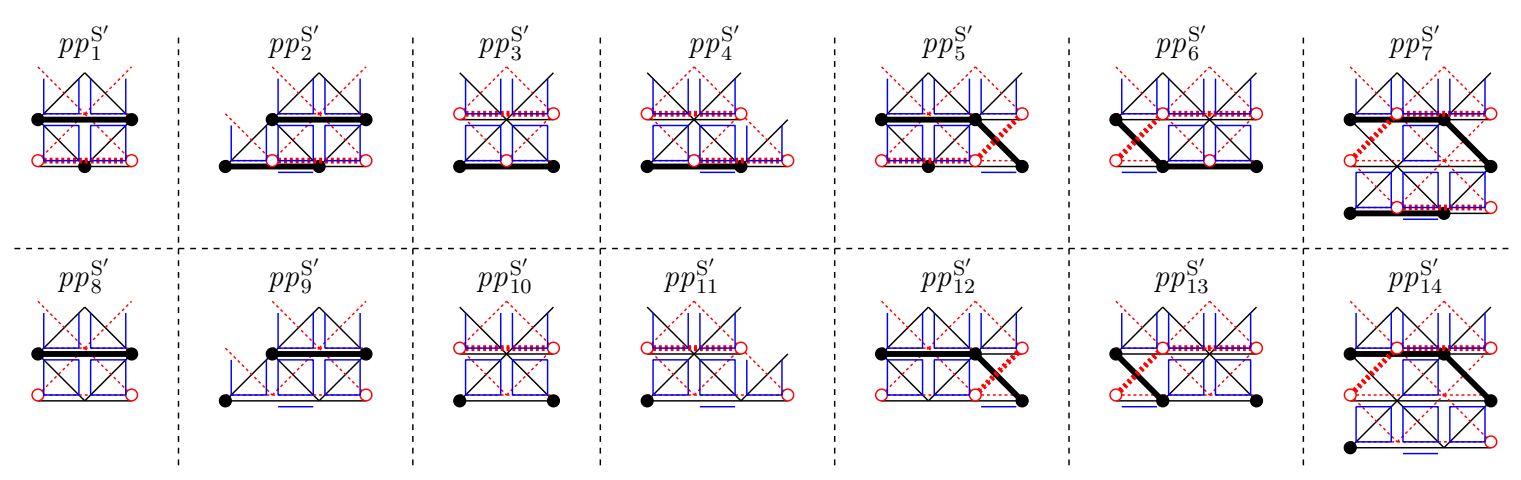

Figure 11: Portions of $\xi^{\prime}$ and $\varphi\left(\xi^{\prime}\right)$ in $\square^{\prime}$.

- Case $K=0$ : Transform $\xi$ into $\varphi(\xi)$ by replacing the piece in $\square$, say $p_{\mathrm{S} \nu}$, with $p_{\mathrm{S}(v+3 \bmod 6)}$.

- Case $K \geq 1$ : Let $\square^{\prime} \subset \mathbb{R}^{2}$ be the set which minimally contains $\square$ and the portion whose form is any of $p p_{1, \ldots, 14}^{\mathrm{S}}$.

(i) Transform $\xi$ into $\xi^{\prime}$ by replacing the portion in $\square^{\prime}$, say $p p_{\nu}^{\mathrm{S}}$, with $p p_{\nu}^{\mathrm{S}^{\prime}}$.

(ii) Transform $\varphi\left(\xi^{\prime}\right)$ into $\varphi(\xi)$ by replacing the portion in $\square^{\prime}$, whose form is $p p_{(\nu+7 \bmod 14)}^{\mathrm{S}^{\prime}}$, with $p p_{(\nu+7 \bmod 14)}^{\mathrm{S}}$.

For example, the non-intersecting but sparse configuration in Figure 7 (the right one) is transformed by $\varphi$ into that in Figure 12, and vice versa. This $\varphi$ is an involution on $\Xi^{\prime}\left(S_{n}, T_{n, i}\right) \backslash \widetilde{\Xi}\left(S_{n}, T_{n, i}\right)$ satisfying the equalities (12), which is easily validated by induction with respect to $K \geq 0$, by using the definition (3) with (6) of weight. Note that $\xi^{\prime}$ in Definition 1 is a non-intersecting configuration which is dense in $\{0 \leq y<K-\kappa\} \cap H_{n}$ and is sparse in $\{K-\kappa \leq y<K-\kappa+1\} \cap H_{n}$, where $\kappa=2$ if the portion of $\xi$ in $\square^{\prime}$ is any of $p p_{7,14}^{\mathrm{S}}$ and $\kappa=1$ otherwise. However it is worth to show how to validate the first equality of (12) between signatures for $\xi \in \Xi^{\prime}\left(S_{n}, T_{n, i}\right) \backslash \widetilde{\Xi}\left(S_{n}, T_{n, i}\right)$ which is sparse in $\{0 \leq y<1\} \cap H_{n}$. Suppose that, for example, $\xi$ has the piece $p_{\mathrm{S} 1}$ in $\square=[2 j, 2 j+2] \times[0,1)$. Then $\xi$ has a path $\omega_{\bar{s}}$ of the form $[\bar{s}, \ldots,(2 j+2,0),(2 j, 0), \ldots, \bar{t}], \bar{s} \geq(2 j+2,0)$ and $\bar{t} \leq(2 j, 0)$, on $G^{-}$and an 


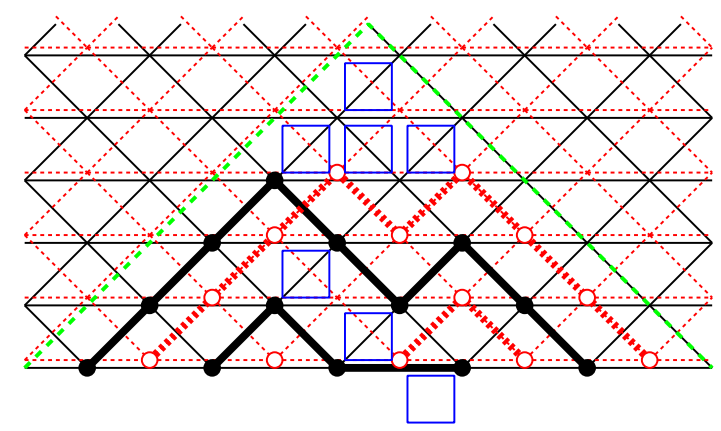

Figure 12: The non-intersecting but sparse configuration to which the right one in Figure 7 is mapped by the involution $\varphi$.

empty path $\omega_{(2 j, 0)}=[(2 j+1,0)]$ on $G^{+}$, for which $\sigma_{\xi}(\bar{s})=\bar{t}$ and $\sigma_{\xi}((2 j, 0))=(2 j+2,0)$. Note that $\varphi(\xi)$ is obtained from $\xi$ only by dividing $\omega_{\bar{s}}$ into two paths $[\bar{s}, \ldots,(2 j+2,0)]$ and $[(2 j, 0), \ldots, \bar{t}]$ by deleting its horizontal edge $((2 j+2,0),(2 j, 0)) \in H_{0}^{-}$and removing the empty path $\omega_{(2 j, 0)}$. Thus $\sigma_{\varphi(\xi)}(\bar{s})=(2 j+2,0), \sigma_{\varphi(\xi)}((2 j, 0))=\bar{t}$ and $\sigma_{\varphi(\xi)}(s)=\sigma_{\xi}(s)$ for the other $s \in S_{n} \backslash\{(2 j, 0), \bar{s}\}$. Hence, since the definition (11), we have the equality. The other cases can be validated similarly.

We have proven Lemma 6.

\subsection{The bijection induced from a non-intersecting and dense configuration}

Using Proposition 8, we may explicitly show the bijection induced from a non-intersecting and dense configuration of Schröder paths, and prove Lemma 7.

Let $\xi \in \widetilde{\Xi}\left(S_{n}, T_{n, i}\right)$ be a non-intersecting and dense configuration, where $n \geq 1$ and $0 \leq i \leq n$. Since Proposition 8, the portion of $\xi$ in $\{0 \leq y<n\} \cap H_{n}$ consists of the pieces $p_{1,2,2^{\prime}, 3,4,4^{\prime}, \mathrm{U}, \mathrm{U}^{\prime}, \mathrm{L} 1, \mathrm{~L} 2, \mathrm{~L} 2^{\prime} \mathrm{R} 1, \mathrm{R} 1^{\prime}, \mathrm{R} 2}$ in Figure 8.

Claim 13. $\xi$ has in each $\{k \leq y<k+1\} \cap H_{n}, 0 \leq k \leq n-1$, no pieces of $p_{3,4,4^{\prime} \mathrm{L} 2, \mathrm{~L} 2^{\prime}, \mathrm{R} 2}$ in the left of each of $p_{1,2,2^{\prime}, \mathrm{L} 1, \mathrm{R} 1, \mathrm{R} 1^{\prime}}$.

Proof. Suppose that $\xi$ does not follow the claim in $\{k \leq y<k+1\} \cap H_{n}$. Then, since Proposition 8, it has in $\{k<y<k+2\} \cap H_{n}$ at least one portion whose form is any in Figure 13. Thus, by induction, $\xi$ does not follow the claim also in $\{n-1 \leq y<n\} \cap$

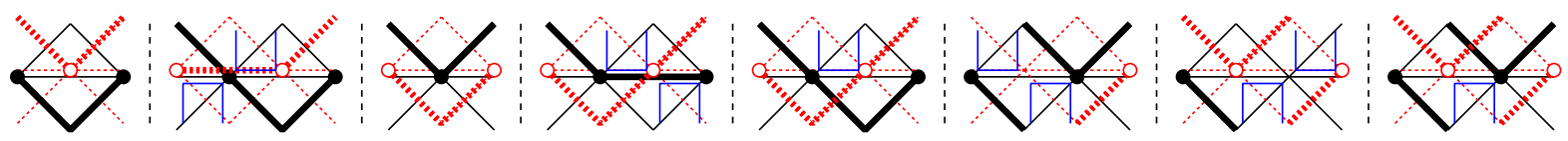

Figure 13: Portions should appear in a non-intersecting and dense configuration if it did not follow Claim 13. 
$H_{n}$, which is a contradiction, for the portion of $\xi$ in there is either $p_{2^{\prime}} p_{\mathrm{L} 2^{\prime}}, p_{2^{\prime}} p_{\mathrm{U}^{\prime}} p_{4^{\prime}}$ or $p_{\mathrm{R} 1^{\prime}} p_{4^{\prime}}$.

Using this claim we may prove Lemma 7 as follows. Let us divide the sources and sinks as $S_{n}=S^{-} \cup S^{+}$and $T_{n, i}=\sigma_{\xi}\left(S^{-}\right) \cup \sigma_{\xi}\left(S^{+}\right)$so that $s \in S^{-}$if and only if the path froward $s$ goes on $G^{-}$(namely iff $s \geq \sigma_{\xi}(s)$ ). Note that $\xi$ has a path on $G^{-}$ (resp. on $G^{+}$) froward $(2 j, 0)$ if and only if it has $p_{3}$ (resp. $\left.p_{1}\right)$ in $[2 j, 2 j+1] \times[0,1)$, any of $p_{\mathrm{L} 2, \mathrm{~L} 2^{\prime}}\left(\operatorname{resp} . p_{\mathrm{L} 1}\right)$ in $([2 j, 2 j+2] \times[0,1)) \cap H_{n}$, or $p_{\mathrm{R} 2}\left(\right.$ resp. any of $\left.p_{\mathrm{R} 1, \mathrm{R} 1^{\prime}}\right)$ in $([2 j-1,2 j+1] \times[0,1)) \cap H_{n}$. Similarly note that $\xi$ has a path on $G^{-}$(resp. on $\left.G^{+}\right)$toward $(2 j, 0)$ if and only if it has any of $p_{2,2^{\prime}}\left(\operatorname{resp} . p_{4,4^{\prime}}\right)$ in $([2 j-1,2 j] \times[0,1)) \cap H_{n}$. Thus, since Claim 13, we have $\min S^{-}>\max S^{+}, \min S^{-} \geq \max \sigma_{\xi}\left(S^{-}\right), \max S^{+}<\min \sigma_{\xi}\left(S^{+}\right)$ and $\max \sigma_{\xi}\left(S^{-}\right)<\min \sigma_{\xi}\left(S^{+}\right)$. Here $\sigma_{\xi}$ must be monotone decreasing respectively in $S^{-}$ and in $S^{+}$since $\xi$ is non-intersecting, and hence it is in the entire $S_{n}$.

We have proven Lemma 7.

\section{A correspondence between non-intersecting and dense configurations of Schröder paths and Favard- LBP paths}

The remaining task to prove Theorem 1 is to clarify a correspondence between nonintersecting and dense configurations of Schröder paths and Favard-LBP paths, where the former interpret determinants of moments by Theorem 5 and the latter do LBPs by Theorem 4.

Theorem 14. Let $n \geq 0$ and $0 \leq i \leq n$. There exists such a bijection $\psi: \widetilde{\Xi}\left(S_{n}, T_{n, i}\right) \rightarrow$ $\Omega_{n, i}^{\mathrm{F}}$ that, for each $\xi \in \widetilde{\Xi}\left(S_{n}, T_{n, i}\right)$, the equality of weight

$$
\left(\prod_{k=0}^{n-1} \alpha_{k}\right) \frac{w(\xi)}{w\left(\xi_{n, n}\right)}=w(\psi(\xi))
$$

holds, where $\xi_{n, n}$ is the unique non-intersecting and dense configuration of Schröder paths in $\widetilde{\Xi}\left(S_{n}, T_{n, n}\right)$.

This section is devoted to prove this theorem.

\subsection{A structure of a non-intersecting and dense configuration}

Before constructing a bijection, we clarify more explicit structure of a non-intersecting and dense configuration of Schröder paths. Since Proposition 8, referring Claims 11 and 13 , we have the following.

Proposition 15. Let $\xi \in \widetilde{\Xi}\left(S_{n}, T_{n, i}\right)$ be a non-intersecting and dense configuration, where $n \geq 1$ and $0 \leq i \leq n$. Then, 
- we have on $\xi$ two neighboring sparse squares $(2 j+k-1,2 j+k) \times(k-1, k)$ and $(2 j+k-2,2 j+k-1) \times(k, k+1)($ resp. $(2 j+k, 2 j+k+1) \times(k, k+1))$, if and only if the portion of $\xi$ in $\{k \leq y<k+1\} \cap H_{n}$ is the piece

$$
p p_{n, k, j}^{\mathrm{L}}= \begin{cases}p_{2^{\prime}} \underbrace{\left(p_{1} p_{2} \cdots\right)}_{2 j-2 \text { pieces }} p_{\mathrm{L} 1} \underbrace{\left(p_{1} p_{2} \cdots\right)}_{m-2 j} \underbrace{\left(\cdots p_{4} p_{3}\right)}_{m-1} p_{4^{\prime}}, & 1 \leq j \leq\left\lfloor\frac{m}{2}\right\rfloor, \\ p_{2^{\prime}} \underbrace{\left(p_{1} p_{2} \cdots\right)}_{m-1 \text { pieces }} \underbrace{\left(\cdots p_{3} p_{4}\right)}_{2 j-m-1} p_{\mathrm{L} 2} \underbrace{\left(\cdots p_{4} p_{3}\right)}_{2 m-2 j-1} p_{4^{\prime}}, & \left\lfloor\frac{m}{2}\right\rfloor+1 \leq j \leq m-1, \\ p_{2^{\prime}} \underbrace{\left(p_{1} p_{2} \cdots\right)}_{m-1 \text { pieces }} \underbrace{\left(\cdots p_{3} p_{4}\right)}_{m-1} p_{\mathrm{L} 2^{\prime}}, & j=m\end{cases}
$$

(resp.

$$
p p_{n, k, j}^{\mathrm{R}}= \begin{cases}p_{\mathrm{R} 1^{\prime}} \underbrace{\left(p_{2} p_{1} \cdots\right)}_{m-1 \text { pieces }} \underbrace{\left(\cdots p_{4} p_{3}\right)}_{m-1} p_{4^{\prime}}, & j=0, \\ p_{2^{\prime}} \underbrace{\left(p_{1} p_{2} \cdots\right)}_{2 j-1 \text { pieces }} p_{\mathrm{R} 1} \underbrace{\left(p_{2} p_{1} \cdots\right)}_{m-2 j-1} \underbrace{\left(\cdots p_{4} p_{3}\right)}_{m-1} p_{4^{\prime}}, & 1 \leq j \leq\left\lceil\frac{m}{2}\right\rceil-1, \\ p_{2^{\prime}} \underbrace{\left(p_{1} p_{2} \cdots\right)}_{m-1 \text { pieces }} \underbrace{\left(\cdots p_{4} p_{3}\right)}_{2 j-m} p_{\mathrm{R} 2} \underbrace{\left(\cdots p_{4} p_{3}\right)}_{2 m-2 j-2} p_{4^{\prime}}, & \left.\left\lceil\frac{m}{2}\right\rceil \leq j \leq m-1\right),\end{cases}
$$

- we have on $\xi$ those $(2 j+k-1,2 j+k) \times(k-1, k)$ and $(2 j+k-1,2 j+k) \times(k+1, k+2)$, if and only if the portions of $\xi$ in $\{k \leq y<k+1\} \cap H_{n}$ and in $\{k+1 \leq y<k+2\} \cap H_{n}$ are the pieces

$$
p p_{n, k, j}^{\mathrm{U}}= \begin{cases}p_{2^{\prime}} \underbrace{\left(p_{1} p_{2} \cdots\right)}_{2 j-1 \text { pieces }} p_{\mathrm{U}} \underbrace{\left(p_{1} p_{2} \cdots\right)}_{m-2 j} \underbrace{\left(\cdots p_{4} p_{3}\right)}_{m-1} p_{4^{\prime}}, & 1 \leq j \leq\left\lfloor\frac{m}{2}\right\rfloor, \\ p_{2^{\prime}} \underbrace{\left(p_{1} p_{2} \cdots\right)}_{m-1 \text { pieces }} \underbrace{\left(\cdots p_{4} p_{3}\right)}_{2 j-m} p_{\mathrm{U}} \underbrace{\left(\cdots p_{4} p_{3}\right)}_{2 m-2 j-1} p_{4^{\prime}}, & \left\lceil\frac{m}{2}\right\rceil \leq j \leq m-1\end{cases}
$$

and

$$
p p_{n, k+1, j}^{\mathrm{U}^{\prime}}= \begin{cases}p_{2^{\prime}} \underbrace{\left(p_{1} p_{2} \cdots\right)}_{2 j-2 \text { pieces }} p_{\mathrm{U}^{\prime}} \underbrace{\left(p_{2} p_{1} \cdots\right)}_{m-2 j} \underbrace{\left(\cdots p_{4} p_{3}\right)}_{m-2} p_{4^{\prime}}, \quad 1 \leq j \leq\left\lfloor\frac{m}{2}\right\rfloor, \\ p_{2^{\prime}} \underbrace{\left(p_{1} p_{2} \cdots\right)}_{m-2 \text { pieces }} \underbrace{\left(\cdots p_{3} p_{4}\right)}_{2 j-m} p_{\mathrm{U}^{\prime}} \underbrace{\left(\cdots p_{4} p_{3}\right)}_{2 m-2 j-2} p_{4^{\prime}}, & \left\lceil\frac{m}{2}\right\rceil \leq j \leq m-1,\end{cases}
$$

respectively,

where $m=n-k$.

For example, the configuration in Figure 6 is obtained by gluing the pieces $p p_{5,0,3}^{\mathrm{L}}, p p_{5,1,2}^{\mathrm{L}}$, $p p_{5,2,1}^{\mathrm{U}}, p p_{5,3,1}^{\mathrm{U}^{\prime}}$ and $p p_{5,4,0}^{\mathrm{R}}$ from bottom to top. Let us define by (3) the weight of a piece 
$F$ of a configuration of Schröder paths. Then the weight of these pieces is given since (3) with (6) by

$$
\begin{aligned}
& w\left(p p_{n, k, j}^{\mathrm{L}}\right)=\frac{\left(\beta_{k+1}\right)^{n-k-1}}{\left(\alpha_{k}\right)^{n-k-1}\left(\gamma_{k}\right)^{n-k}}, \quad w\left(p p_{n, k, j}^{\mathrm{R}}\right)=\frac{\left(\beta_{k+1}\right)^{n-k-1}}{\left(\alpha_{k}\right)^{n-k}\left(\gamma_{k}\right)^{n-k-1}}, \\
& w\left(p p_{n, k, j}^{\mathrm{U}}\right)=\frac{\left(\beta_{k+1}\right)^{n-k}}{\left(\alpha_{k}\right)^{n-k}\left(\gamma_{k}\right)^{n-k}}, \quad w\left(p p_{n, k+1, j}^{\mathrm{U}^{\prime}}\right)=\frac{\left(\beta_{k+2}\right)^{n-k-2}}{\left(\alpha_{k+1}\right)^{n-k-1}\left(\gamma_{k+1}\right)^{n-k-1}} .
\end{aligned}
$$

\subsection{A bijection between a non-intersecting and dense configu- ration and a Favard-LBP path}

Since Proposition 15 , we can define a map $\psi: \widetilde{\Xi}\left(S_{n}, T_{n, i}\right) \rightarrow \Omega_{n, i}^{\mathrm{F}}$, where $n \geq 0$ and $0 \leq i \leq n$, as follows.

Definition 2 (Bijection $\left.\psi: \widetilde{\Xi}\left(S_{n}, T_{n, i}\right) \rightarrow \Omega_{n, i}^{\mathrm{F}}\right)$. On a given non-intersecting and dense configuration $\xi \in \widetilde{\Xi}\left(S_{n}, T_{n, i}\right)$ of Schröder paths, where $n \geq 0$ and $0 \leq i \leq n$, we draw a Favard-LBP path $\psi(\xi) \in \Omega_{n, i}^{\mathrm{F}}$ as follows.

(i) Put a vertex at the center of every sparse square on $\xi$.

(ii) Connect every two neighboring vertices with an edge.

For example, we draw on the configuration in Figure 6 as in Figure 14. Note that the pieces $p p_{n, k, j}^{\mathrm{L}}, p p_{n, k, j}^{\mathrm{R}}$ and $p p_{n, k, j}^{\mathrm{U}}$ in a configuration induce the edges $((2 j+k-1 / 2, k-$ $1 / 2),(2 j+k-3 / 2, k+1 / 2)) \in L_{k}^{\mathrm{F}},((2 j+k-1 / 2, k-1 / 2),(2 j+k+1 / 2, k+1 / 2)) \in R_{k}^{\mathrm{F}}$ and $((2 j+k-1 / 2, k-1 / 2),(2 j+k-1 / 2, k+3 / 2)) \in U_{k+1}^{\mathrm{F}}$ of a Favard-LBP path, respectively. This $\psi$ is clearly injective, while it is surjective since, for example, the piece $p p_{n, k, j}^{\mathrm{L}}(0 \leq k \leq n-2)$ in $\{k \leq y<k+1\} \cap H_{n}$ fits every piece in $\{k+1 \leq y<k+2\} \cap H_{n}$ of $p p_{n, k+1, j-1}^{\mathrm{L}}$ (if $2 \leq j \leq n-k$ ), $p p_{n, k+1, j-1}^{\mathrm{R}}$ (if $1 \leq j \leq n-k-1$ ) and $p p_{n, k+1, j-1}^{\mathrm{U}}$ (if $2 \leq j \leq n-k-1)$. Thus $\psi$ is bijective.

The bijection $\psi$, with Proposition 15, tells us the following. The set $\Omega_{n, n}^{\mathrm{F}}$ of FavardLBP paths going from $(n-1 / 2,-1 / 2)$ to $(n-1 / 2, n-1 / 2)$ contains the unique path $\omega_{n, n}^{\mathrm{F}}$

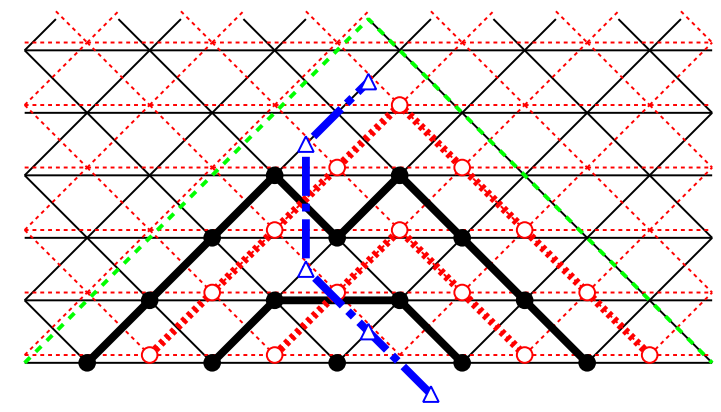

Figure 14: The Favard-LBP path in $\Omega_{5,3}^{\mathrm{F}}$ drawn by $\psi$ on the configuration of Schröder paths in Figure 6. (Sparse squares are omitted.) 
only of left-diagonal edges. Thus the set $\widetilde{\Xi}\left(S_{n}, T_{n, n}\right)$ contains the unique non-intersecting and dense configuration $\xi_{n, n}=\psi^{-1}\left(\omega_{n, n}^{\mathrm{F}}\right)$ consisting of the pieces $p p_{n, 0, n}^{\mathrm{L}}, p p_{n, 1, n-1}^{\mathrm{L}}, \ldots$, $p p_{n, n-1,1}^{\mathrm{L}}$ from bottom to top. (Similarly, $\Omega_{n, 0}^{\mathrm{F}}$ contains the unique $\omega_{n, 0}^{\mathrm{F}}$ only of rightdiagonal edges, and $\widetilde{\Xi}\left(S_{n}, T_{n, 0}\right)$ does the unique $\xi_{n, 0}=\psi^{-1}\left(\omega_{n, 0}^{\mathrm{F}}\right)$ consisting of $p p_{n, 0,0}^{\mathrm{R}}$, $p p_{n, 1,1}^{\mathrm{R}}, \ldots, p p_{n, n-1, n-1}^{\mathrm{R}}$ from bottom to top.) See Figure 15 for example. Thus, since (14) leads

$$
\alpha_{k} \frac{w\left(p p_{n, k, j}^{\mathrm{X}}\right)}{w\left(p p_{n, k, n-k}^{\mathrm{L}}\right)}= \begin{cases}\alpha_{k}, & \mathrm{X}=\mathrm{L} \\ \gamma_{k}, & \mathrm{X}=\mathrm{R} \\ \beta_{k+1}, & \mathrm{X}=\mathrm{U} \\ 1, & \mathrm{X}=\mathrm{U}^{\prime}\end{cases}
$$

we have, with (3) with (6) and (9), the equality (13).

We have proven Theorem 14.

\section{A proof of Theorem 1}

In this final section, we accomplish our purpose, that is, we give a combinatorial proof of Theorem 1, using Theorems 5 and 14 for configurations of Schröder paths and Favard-LBP paths.

We found in Section 4 that $\widetilde{\Xi}\left(S_{n}, T_{n, n}\right)=\left\{\xi_{n, n}\right\}$ and $\widetilde{\Xi}\left(S_{n}, T_{n, 0}\right)=\left\{\xi_{n, 0}\right\}$, which implies through Theorem 5

$$
\Delta_{n}^{(0)}=\Delta_{n, n}=(-1)^{\frac{n(n-1)}{2}}\left(\mu_{0} \gamma_{0}\right)^{n} w\left(\xi_{n, n}\right), \quad \Delta_{n}^{(1)}=\Delta_{n, 0}=(-1)^{\frac{n(n-1)}{2}}\left(\mu_{0} \gamma_{0}\right)^{n} w\left(\xi_{n, 0}\right) .
$$

Hence, since the definition (3) with (6) of weight, both of the Toeplitz determinants $\Delta_{n}^{(0)}$ and $\Delta_{n}^{(1)}$ are nonzero. Moreover, using these equalities, we may easily derive the equalities (2a), for which the equalities, for $n \geq 0$,

$$
\frac{w\left(\xi_{n+1, n+1}\right)}{w\left(\xi_{n, n}\right)}=w\left(\hat{\omega}_{-n}\right)=\left(\gamma_{0}\right)^{-1} \prod_{k=1}^{n} \frac{\beta_{k}}{\gamma_{k-1} \gamma_{k}}, \quad \frac{w\left(\xi_{n+1,0}\right)}{w\left(\xi_{n, 0}\right)}=w\left(\hat{\omega}_{n+1}\right)=\left(\alpha_{0}\right)^{-1} \prod_{k=1}^{n} \frac{\beta_{k}}{\alpha_{k-1} \alpha_{k}}
$$
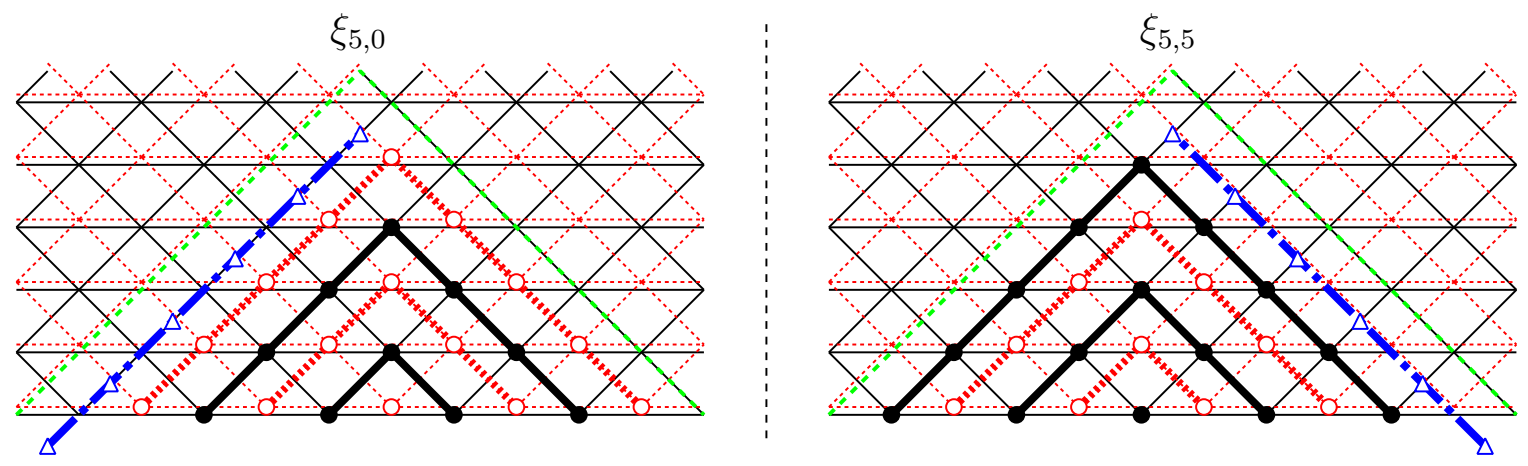

Figure 15: The unique configurations $\xi_{5,0} \in \widetilde{\Xi}\left(S_{5}, T_{5,0}\right)$ and $\xi_{5,5} \in \widetilde{\Xi}\left(S_{5}, T_{5,5}\right)$, and the corresponding Favard-LBP paths $\omega_{5,0}^{\mathrm{F}}=\psi\left(\xi_{5,0}\right)$ and $\omega_{5,5}^{\mathrm{F}}=\psi\left(\xi_{5,5}\right)$ on them. 
shall be helpful, where $\hat{\omega}_{\ell} \in \Omega_{\ell}$ is the unique Schröder path with exactly one peak. (See Figure 15.) Finally, through Theorem 5, we can rewrite the equality (13) in terms of determinants into

$$
\left(\prod_{k=0}^{n-1} \alpha_{k}\right) \frac{\Delta_{n, i}}{\Delta_{n}^{(0)}}=\sum_{\omega^{\mathrm{F}} \in \Omega_{n, i}^{\mathrm{F}}} w\left(\omega^{\mathrm{F}}\right)
$$

by summing the equality over $\xi \in \widetilde{\Xi}\left(S_{n}, T_{n, i}\right)$, which, through Theorem 4 , immediately leads the determinant representation $(2 \mathrm{~b})$ of the LBPs.

Thus we have completely proven Theorem 1.

\section{References}

[1] J. Bonin, L. Shapiro and R. Simion, Some $q$-analogues of the Schröder numbers arising from combinatorial statistics on lattice paths, J. Statist. Plann. Inference 34 (1993), no. 1, 35-55.

[2] P. Flajolet, Combinatorial aspects of continued fractions, Discrete Math. 32 (1980), no. 2, 125-161.

[3] I. Gessel and G. Viennot, Binomial determinants, paths, and hook length formulae, Adv. in Math. 58 (1985), no. 3, 300-321.

[4] E. Hendriksen and H. van Rossum, Orthogonal Laurent polynomials, Nederl. Akad. Wetensch. Indag. Math. 48 (1986), no. 1, 17-36.

[5] M.E.H. Ismail and D.R. Masson, Generalized orthogonality and continued fractions, J. Approx. Theory 83 (1995), no. 1, 1-40.

[6] W.B. Jones and W.J. Thron, Survey of continued fraction methods of solving moment problems and related topics, Analytic theory of continued fractions (Loen, 1981), pp. 4-37, Lecture Notes in Math., 932, Springer, Berlin-New York, 1982.

[7] S. Kamioka, A combinatorial representation with Schröder paths of biorthogonality of Laurent biorthogonal polynomials, Electron. J. Combin. 14 (2007), no. 1, Research Paper 37, 22 pp. (electronic).

[8] N.J.A. Sloane, The On-Line Encyclopedia of Integer Sequences, published electronically at www.research.att.com/ ${ }^{\sim}$ njas/sequences/.

[9] G. Viennot, Une théorie combinatoire des polynômes orthogonaux généraux, Notes de conférences données à l'UQAM, Montréal, 1983.

[10] L. Vinet and A. Zhedanov, Spectral transformations of the Laurent biorthogonal polynomials. I. q-Appel polynomials, J. Comput. Appl. Math. 131 (2001), no. 1-2, 253-266.

[11] A. Zhedanov, The "classical" Laurent biorthogonal polynomials, J. Comput. Appl. Math. 98 (1998), no. 1, 121-147. 